\title{
A model for the dependence of carbon nanotube length on acid oxidation time
}

\author{
Gavin A. Forrest and Andrew J. Alexander* \\ School of Chemistry, University of Edinburgh, West Mains Road, Edinburgh, EH9 3JJ \\ *email: andrew.alexander@ed.ac.uk
}

\section{Supporting Information}

\section{Analysis of TEM images}

Analysis of the distribution of nanotube lengths observed was performed on TEM images using image analysis software (Image J, National Institues of Health, USA). The TEM micrographs were randomly selected. Samples were taken from three $1 \mu \mathrm{m}$ by $1 \mu \mathrm{m}$ regions. A typical TEM image of treated nanotubes and amorphous carbon (at 4 hours) is shown below (scale bar $=200 \mathrm{~nm}$ ). The sample contains individual nanotubes, or bundles of nanotubes, surrounded by amorphous carbon resulting from the oxidation process. The imaging software was used to continually adjust contrast and pixel levels of the images, and tubes and tube-bundles were visually traced using the software, which then calculated and output the lengths, accounting for curved features. Nanotubes longer than 1 micron would have been included if they were curved and so fitted into the sample area. According to CNI, the as-purchased HiPco tubes are $0.3-1.0 \mu \mathrm{m}$ in length, so these tubes are expected to be relatively few: this agrees with our own analysis of the as-purchased sample (at 0 hours).

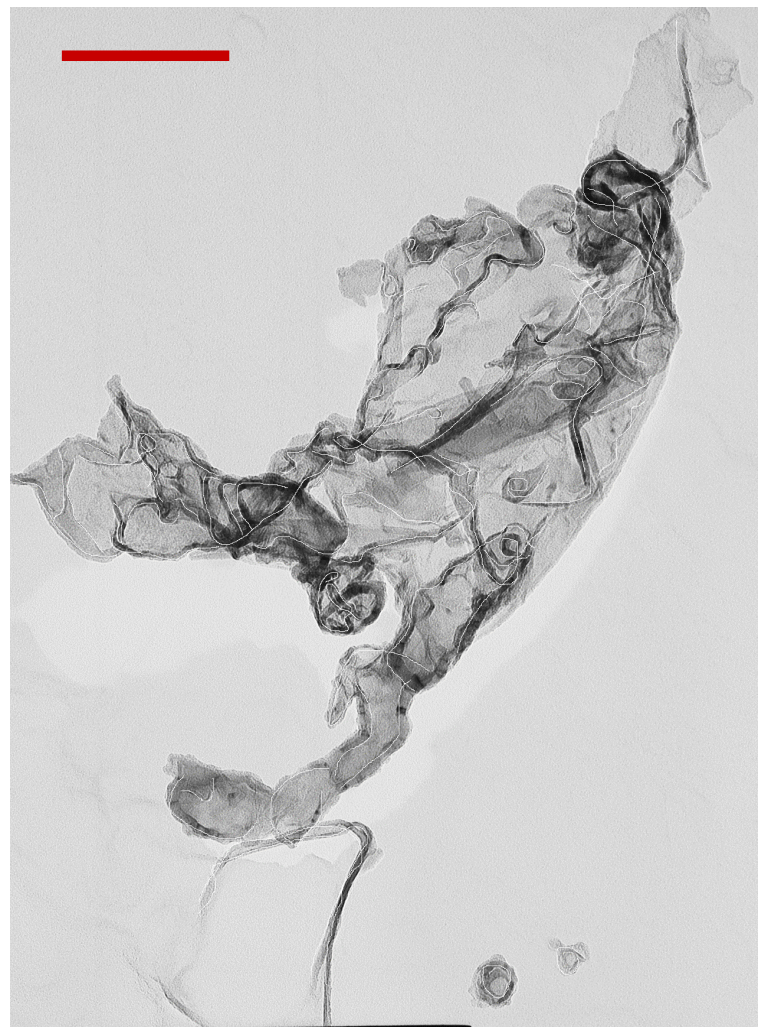




\section{Polishing of tubes with Piranha solution}

See Section 3, and Figures 3 (a) and (b) for oxidatively shortened tubes that have been polished with piranha solution. The Raman spectrum for the polished tubes is given below, in comparison to the as-purchased HiPco tubes prior to oxidative treatment (0 hours sample). The final $\mathrm{D} / \mathrm{G}$ ratios were 0.12 (HiPco) and 0.15 ( $2 \mathrm{~h}$ treated, piranha)

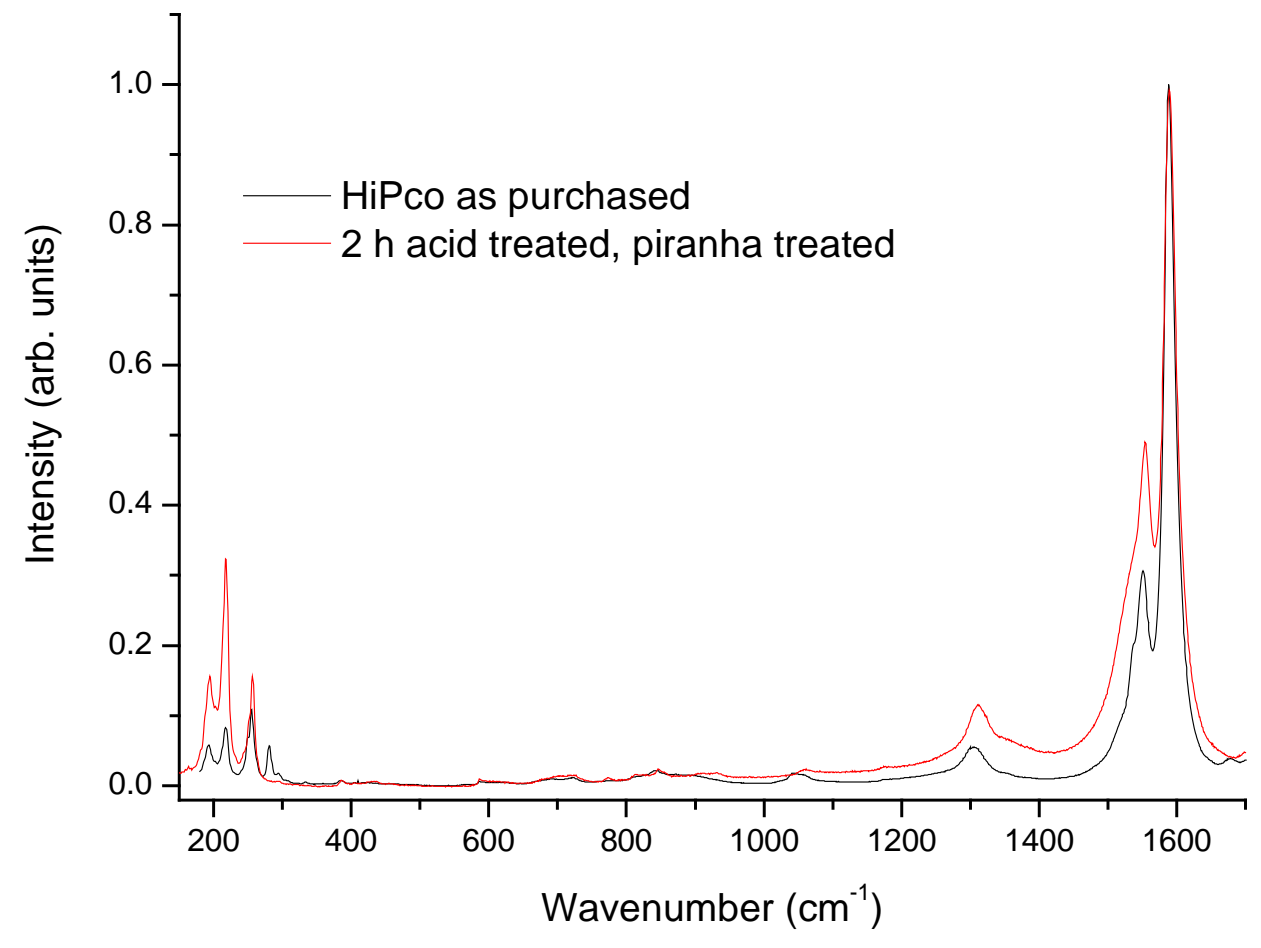

